\begin{tabular}{ccc}
\hline International Journal of Engineering \& Technology, $7(2.33)(2018) 603-606$ \\
SPC & Website www.sciencepubco.com/index.php/IJET \\
Research paper & Technology \\
\hline
\end{tabular}

\title{
PET SFCM image segmentation for alzheimer's disease
}

\author{
Dr. A. Meenakabilan ${ }^{1 *}$, Agathiyan $\mathbf{K}^{2}$ \\ ${ }^{I}$ Assistant Professor, Dept. of CSE, Sri Sairam Engineering College, Chennai \\ ${ }^{2}$ UG Scholar, AITEM, Amity University, Noida \\ *Corresponding author E-mail: kabimeena2@gmail.com
}

\begin{abstract}
Medical images are known to capture the human body in both anatomical and functional view. These images are interpreted with expert domain for clinical analysis. Here, the selection of image sample plays a fundamental role. However, doctors need to manually obtain this process. But, in order to get similarity between the samples automation is definitely required as it reduces the computation time. So, the automation process should be knowledge based to get better results. This paper highlights the knowledge based automation of medical image sample analysis. It presents a significant assessment of PET - SFCM approach for the segmentation of functional medical images which is considered as the value of neighboring pixels in spatial correlation. Here, the proposed method is used to apply the decision support strategy to identify the effective samples from the huge data collection. The proposed algorithm is implemented in Matlab 7.0. The obtained results were analyzed and compared with other two clustering approaches known as K-Means and Fuzzy C-Means. The resultant images encourage the identification and an evaluation of treatment response in a set of oncological constraints.
\end{abstract}

Keywords: Knowledge Base; PET Scan Image; Alzheimer's Disease; K-Means; Fuzzy C-Means; SFCM Clustering.

\section{Introduction}

Image diagnosis is an important task in medical environment. Digital image processing refers to processing of digitized images through computers and finds varieties of application in the most assorted areas of research, science and technology. In the recent years, the most important diagnostic tool in medical application is medical imaging. Computed Tomography (CT), Magnetic Resonance Image (MRI), Positron Emission Tomography (PET) and other imaging modalities have greatly increased the knowledge of anatomical structure and treatment planning. For many years, anatomical imaging known as CT and MRI were used for radiation therapy. Now the dynamic PET image has emerged as a promising approach in modern world for treatment planning. PET and SPECT are commonly known as functional modalities for diagnostic imaging. But, both are to limit unusual tissues because of generally poor spatial determination and high commotion levels. Manual delineation is more time consuming and is device dependent. Due to the cast number of medical images and its size the need of automation has become necessary. Semi or fully automated segmentation methods are used to improve the reproducibility. Most of the researchers present the status of semi-robotized and automated techniques for the classification of anatomical therapeutic images. Brain is the most interesting organ in the human body. Based on region of interest, the standardized uptake value is calculated by clinicians for diagnosis. The clinical diagnosis could be improved by various methods of pattern recognition such as segmentation of PET data. In segmentation, the clustering algorithm provides a solution to find out the region of interest depends on the voxel classification.

Clustering is known as the most important unsupervised learning approach in data mining process. The process of clustering is equivalent to classification where similar members are grouped together without the need of training samples. It means, clustering approach train themselves using the available data. The arrival of new clustering techniques is used for developing segmentation in various research domains. Clustering algorithm is used to provide the updated centroid value according to the distance between the objects.

Nuclear medicine imaging is known as PET scan, detects the metabolism changes as chemical and physiological. Radioactive tracer element which is injected in the body and the tumors or cancers in the body are identified based on the movements of the tracer element [1]. PET scan images shows the internal formation of tumors and cancer cells by means of the metabolism of the body parts [2]. The rest of this paper is organized as follows: Neuro degenerative disorder such as Alzheimer's disease is described in section 2. In section 3, related work in this field is described. Section 4 presents a description of the proposed method. The implantation results are presented in section 5. The last part describes our conclusion.

\section{Neuro degenerative disorders - alzheimer's disease}

The source of energy for brain is the combination of oxygen and glucose. The variation of blood flow gives many brain disorders like Alzheimer's disease (AD). It is a common dementia, especially senior people. Dementia is a brain disorder refers to serious loss of cognitive functions and affects day to day activities. AD is the most important public health problem in both developing and developed countries [3]. In early $20^{\text {th }}$ Century, German physician, Dr. Alois Alzheimer described the disease. Two main features of AD are plaques and tangles in the brain and the third reason is the loss of connections between nerve cells (neurons). The cause and progression of AD are poorly understood. Increasing age is its single most important risk factor for $\mathrm{AD}$. Genetic predisposition (specific genetic factors) has also been reported to be a risk factor. Women of older age have higher risk than men. Metabolic syndrome and vascular risk factors have a strong association with AD. At the initial 
stage some function of brain (memory, language) is involving. Patients may have difficulties in short-term memory (remembering recent happenings or with known peoples' names). At a later stage, more memory problems than that expected for people of that age. In the more advanced stages of $\mathrm{AD}$, behavioral changes and psychiatric symptoms are usually present. These changes include agitation, paranoia, psychosis, delusions, anxiety and insomnia. Advanced medical imaging techniques are used to aid in ruling out pathological causes or subtypes of dementia.

\section{Related work}

Koon et.al explained the automatic segmented dynamic PET images using clustering approach. The resultant image was validated with phantom study and evaluated with real dynamic PET data of malignant primary brain, right mesial temporal lobe and aggressive mesothelioma in the left lung [4]. Dinesh.E. et. al [5] was extracted different kinds of brain images from functional magnetic resonance image (fMRI), single photon emission computed tomography (SPECT) database and positron emission tomography (PET) images having Alzheimer's disease. Characteristics selection and extraction were done by applying the Fisher Discriminant Ratio (FDR) and Nonnegative Matrix Factorization (NMF) methods. Support Vector Machine based classifier was used to classify the images. In medical image segmentation, clustering algorithm plays a vital role. Chan et.al [6] described a new 3D image segmentation by adaptive K-Means clustering together with knowledge based morphological operation. The proposed methodology applied to segment the volumes of the left ventricles in cardiac CT volumetric images. In paper [7], the author demonstrated the physiological features related with Alzheimer's disease. This model was developed the individualized biomarkers allowing accurate early diagnosis and treatment evaluation and also identified the cause of AD syndrome. Yao et. al [8] concentrated on deformable models to colonic polyp segmentation in CT colonography using Fuzzy clustering algorithm. Shijun Wang et. al [9] presented an overview of machine learning and its applications in radiology. Machine learning is used to identify the complex patterns in various types of radiological data. Chaves et. al [10] explained to determine the known pattern between attributes in functional brain imaging. The author applied Fisher Discriminant Ratio (FDR) for masking to reduce the computational cost which is used to classify relationships among brain areas. In [11], Rajendran et. al segmented tumor region on MRI images by fuzzy clustering with deformable model using gradient vector field as a external force field.

\section{Methods used}

The whole datasets contains a patient of probable Alzheimer's disease having 266 samples. An FDG-PET brain image sample of 45 years is shown in fig. 1 .
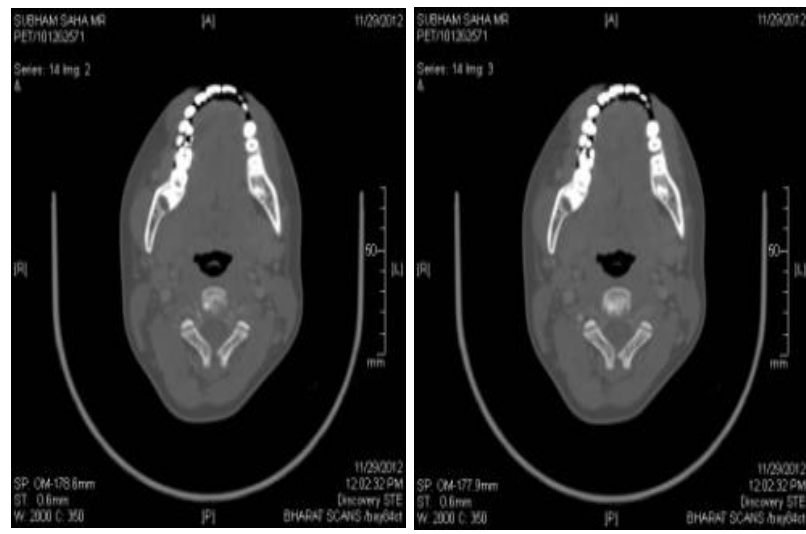

Fig. 1: FDG-PET Images of 45-Year Male Patient.
Though sample is identified the computation process has to be done to get the similar values from the Jaccard Coefficient. A statistic index of Jaccard is used for comparing the sample sets. The two samples are disjoint if the Jaccard index is zero. Better agreement of samples identifies with higher numbers. The goal is to get as close to [1] as possible. This decision process is very effective as it reduced time for sample analysis. Clustering algorithm is used to yield a threshold value for the segmentation process.

The overall system design is shown in Fig.2. The system diagram is divided in to two major classifications. The first part explains the knowledge based sample analysis and the second part explains the cluster based image segmentation. Here, the total number of series is known as $\mathrm{n}$. So, the iteration takes place from the first sample to ' $n$ ' samples. After applying the decision making strategy selective number of samples are filtered. Now, the filtered one is given as an input for the cluster related image segmentation.

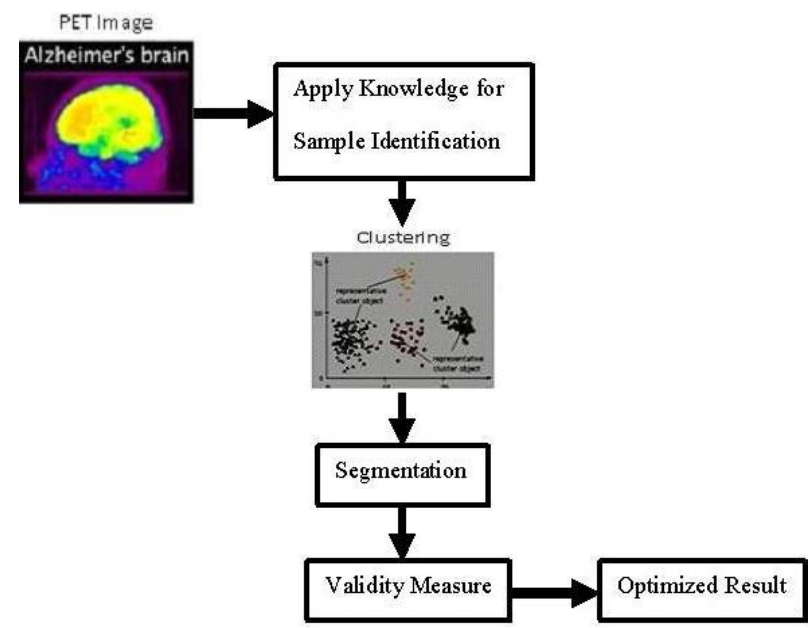

Fig. 2: Knowledge Based System Design.

The proposed clustering algorithm is applied to the filtered sample image and to find the centroid value. Then, based on the centroid value the real PET scan image segmentation is done in our previous work. This paper is explained the decision strategy for PET SFCM. Here, there is no need to submit all the collected input samples in to cluster centroid updation. First, the sample which contains more useful information is to be identified from the number of samples. Then, clustering is applied only for the selected samples and therefore the number of iteration is reduced for segmentation. Fuzzy C-Means is known as a soft clustering method for image segmentation. The conventional FCM is more sensitive to noise and not to incorporate the spatial information in the image. The proposed PET-SFCM is used to improve the performance of conventional one.

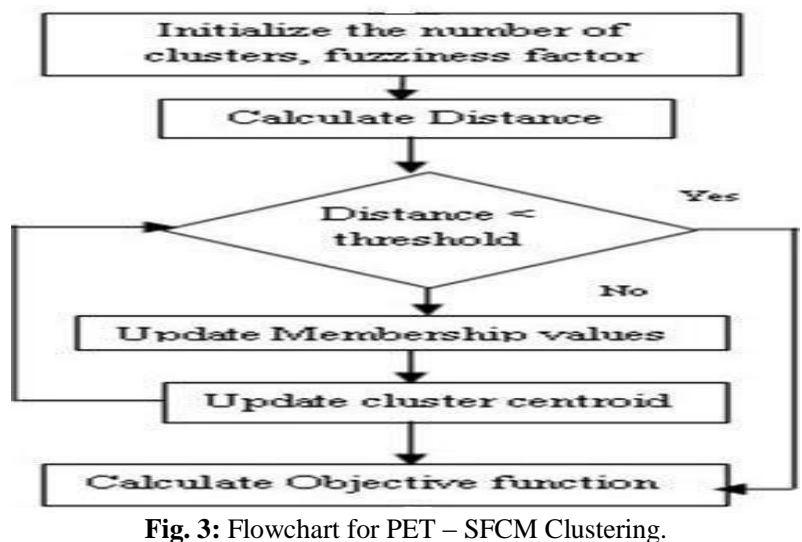

Depends on the degrees of membership, PET-SFCM allows a pixel in more than one cluster. Summation of membership of each data points in the given datasets should be equal to each other. Let $\mathrm{X}=$ $\left\{\mathrm{x}_{1}, \mathrm{x}_{2}, \mathrm{x}_{3} \ldots, \mathrm{x}_{\mathrm{n}}\right\}$ be the set of data points and $\mathrm{C}=\left\{\mathrm{c}_{1}, \mathrm{c}_{2}, \mathrm{c}_{3} \ldots, \mathrm{c}_{\mathrm{n}}\right\}$ 
be the set of centers. The following equations 1 and 2 describe the membership and cluster center updation for each iteration.

$$
\begin{aligned}
& \mu_{\mathrm{ij}}=\frac{1}{\sum_{\mathrm{k}=1}^{\mathrm{c}}(\mathrm{dij} / \mathrm{dik})^{(2 \mathrm{~m}-1)}} \\
& c_{j}=\sum_{i=1}^{n}\left(\frac{\left(\left(\mu_{i j}\right)^{\mathrm{m}} x i\right)}{\left(\mu_{i j}\right)^{\mathrm{m}}}\right)
\end{aligned}
$$

Where,

$\mathrm{d}_{\mathrm{ij}}$ - Distance between $\mathrm{i}^{\text {th }}$ data and $\mathrm{j}^{\mathrm{th}}$ cluster center c - Number of cluster

$\mathrm{m}$ - Fuzziness index

$\mu_{\mathrm{ij}}$ - Membership of $\mathrm{i}^{\text {th }}$ data to $\mathrm{j}^{\text {th }}$ cluster center

$\mathrm{n}$ - Number of data points.

$\mathrm{c}_{\mathrm{j}}-\mathrm{j}^{\text {th }}$ cluster center

Procedure for image segmentation

Step 1: Intialize centroids corresponding to the

Required number of clusters

Step 2: Calculate original centroid

(Call PET - SFCM)

Step 3: Calculate the mask

Step4: Do the segmentation

The above segmentation pseudo code describes the image segmentation using an objective function which is derived from PET SFCM clustering algorithm. After the completion of segmentation process, the segmented image is given as an input for the cluster validity measures. Calculation of the similarity between pairs of clustering is done by cluster measure. None of the metrics shows dominance that could allow considering it as the best metric. The need of clustering is to ascertain the separation between two datasets, which will distinguish the comparability of two elements. This will impact the shape of the clusters, as a few elements might be near each other as per one separation and more remote away as indicated by another. Traditionally Euclidean Distance (ED) is used in clustering however as the choice of other metric in definite cases may be disputable. Distance between two datasets is used to identify the similarity. In general, smaller ED means better similarity and vice versa. It depends on the amount of data and the complexity of the task. So, we have concluded in our experiment [12] the chebyshev distance is always better than those of Euclidean distance and it is suitable for fuzzy clustering also. Here, we considered two basic set of algorithms. One is hard clustering and another one is based on soft clustering.

\section{Results and discussion}

Fig.4 shows a downloaded PET scan image of normal brain and Alzheimer affected one.
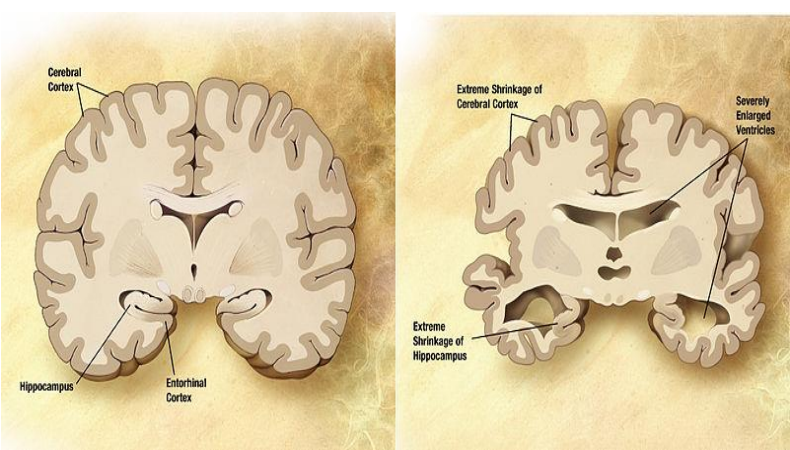

Fig. 4: Normal Brain and Alzheimer Affected Brain.

To examine the validity of the segmentation scheme, a real PET study was taken. Brain PET images were derived from 8 patients. The original size of the image is $598 \times 676$. The data were acquired on an ECAT 931 scanner (CTI/Siemens, Knoxville, TN). Images were reconstructed using filtered back-projection (FBP) with a Hann filter cut off at the Nyquist frequency.Fig.4 shows few samples from ' $n$ ' series.
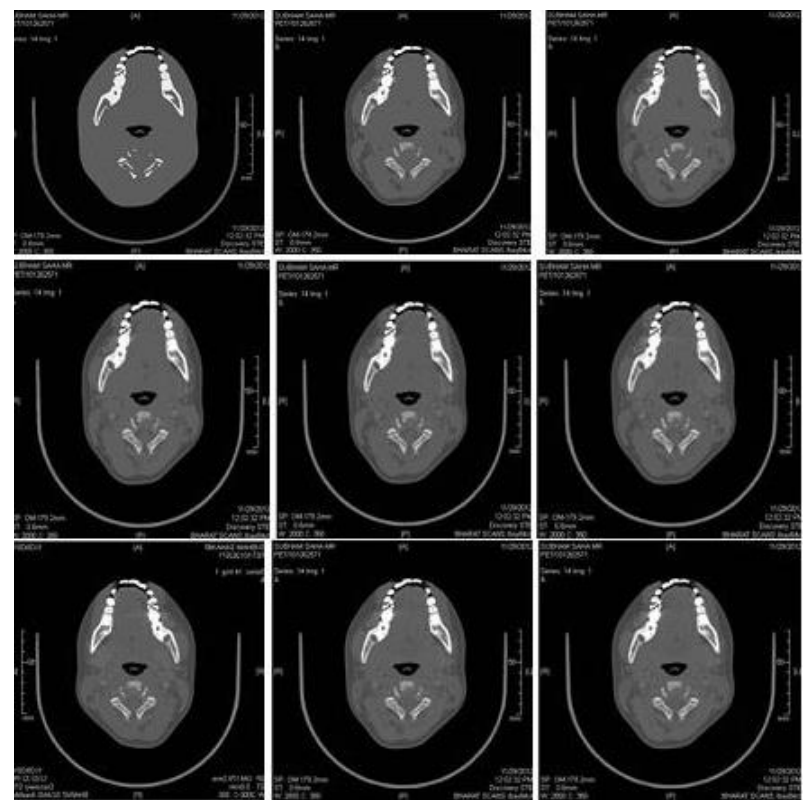

Fig. 5: Series of Real Images from N Samples.

The below Fig. 6.shows the segmented image obtained from KMeans clustering algorithm. The comparison results of PET segmented image from Fuzzy C-Means clustering as shown in fig.7. The proposed model PET-SFCM resultant image is shown in Fig.8.
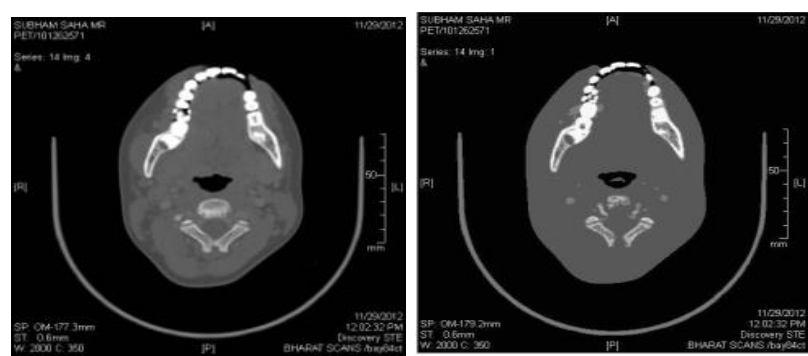

Fig. 6: Segmented Image Obtained from K-Means Clustering.

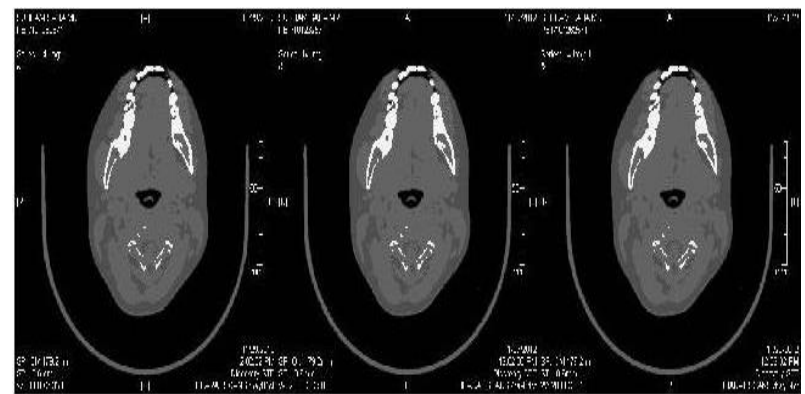

Fig. 7: Segmented Image Obtained from FCM Clustering. 

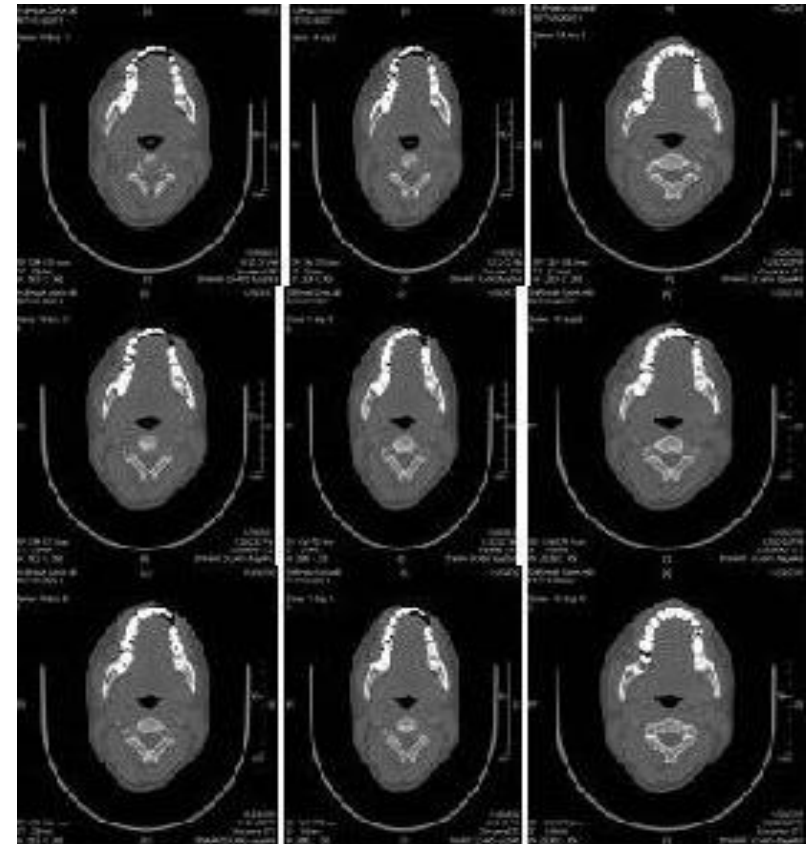

Fig. 8: Segmented Image Obtained from Proposed PET - SFCM Clustering.

The quality of segmentation is evaluating by several methods based on different application domain. The quality of the given segmentation can then be measured by PR index value. Here, precision is calculated as follows

Precision $=\mathrm{TP} /(\mathrm{TP}+\mathrm{FP})$

Where

TP - True positive which produces correct result

FP - False positive which gives an unexpected result

Normally, greater recall increases the possibilities of removing normal cells in negative outcome and all abnormal cells in positive outcome. But, greater precision decreases the possibilities of removing normal cells in positive outcome and all abnormal cells in negative outcome. In our application domain, we concentrated greater precision factor alone. It identified a higher precise value of segmented image as an optimized resultant image.

\section{Conclusion}

This paper is proposed the knowledge based segmentation for Alzheimer's disease. This experiment was made on 265 images from consecutive dynamic sequence of PET images. The image segmentation approach can help physicians automatically select the samples from successive sequence and accurately classify the abnormal pixels in brain images. It effectively reduces human operation time and errors.

\section{References}

[1] Koon-Pong Wong, Dagan Geng, Steven R.Meikle, Michael J.Fulham "Segmentation of Dynamic PET Images Using cluster analysis" IEEE Transactions on nuclear science, Vol. 49, pp.200-207, 2002.

[2] Andreas Hapfelmeier, Jana Schmidt, Marianne Muller, Stefan Kramer "Interpreting PET scans by structured Patient Data: A Data mining case study in dementia Research" IEEE Knowledge and Information Systems, pp.213-222, 2009.

[3] Meena A, Raja K, "K-Means Segmentation of Alzheimer's Disease In Pet Scan Datasets - An Implementation" International Conference on Advances in Signal Processing and Information Technology, Springer, Institute for Computer Sciences, Social Informatics and Telecommunications Engineering - LNICST, ISSN:1867-8211 pp. 158-162, 2012

[4] Segmentation of dynamic PET images using cluster analysis, KoonPong Wong, IEEE Transactions on Nuclear Science, Vol. 49, No.1, $200-207,2002$
[5] Instinctive classification of Alzheimer's disease using FMRI, pet and SPECT images, Dinesh, E. ET. al, 7th International Conference on Intelligent Systems and Control (ISCO), 405 - 409, 2013

[6] Chan, H.-P., Hadjiiski, L., Zhou, C., Sahiner, B., Computer-aided diagnosis of lung cancer and pulmonary embolism in computed tomography - a review. Academic Radiology 15, 535-555, 2008

[7] Generative FDG-PET and MRI Model of Aging and Disease Progression in Alzheimer's Disease, Juergen Dukart, 21st Annual International Conference on Intelligent systems and Molecular Biology, 2013.

[8] Yao, J.H., Miller, M., Franaszek, M., summers, R.M., "Colonic polyp segmentation in CT colonography-based on fuzzy clustering and deformable models", IEEE Transactions on Medical Imaging 23, 1344-1352, 2004.

[9] Shijun Wang, Ronald M. Summers, "Machine learning and radiology", Medical Image Analysis 16, Elsevier, 933-951, 2012

[10] Chaves. R , Ramirez J, J.M. Gorriz, I.A. Illan, "Functional brain image classification using association rules defined over discriminant regions", Pattern Recognition Letters 33, Elsevier ,1666-16, 2012

[11] Rajendran A, Dhanasekaran R, "Fuzzy Clustering and Deformable Model for Tumor Segmentation on MRI Brain Image: A Combined Approach" Procedia Engineering 30, Elsevier, 327 - 333, 2012

[12] Meena A, Raja K, "Cluster Based Performance Analysis of PET Scan Image for Alzheimer's Disease" Advances in Computational Sciences and Technology, ISSN 0973-6107 Volume 6, Number 1 (2013) pp. 81-87 